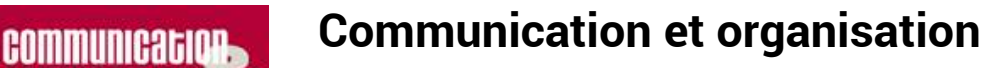

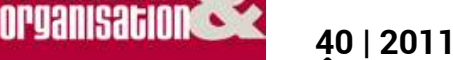

Âges et générations : la communication revisite ses publics

\title{
Oser les cheveux blancs : une stratégie de communication à Bordeaux
}

Entretien réalisé par Agnès Pecolo

\section{Agnès Pecolo}

\section{OpenEdition \\ Journals}

Édition électronique

URL : http://journals.openedition.org/communicationorganisation/3618

DOI : 10.4000/communicationorganisation.3618

ISSN : 1775-3546

Éditeur

Presses universitaires de Bordeaux

\section{Édition imprimée}

Date de publication : 1 décembre 2011

Pagination : 191-193

ISBN : 978-2-86781-745-8

ISSN : $1168-5549$

\section{Référence électronique}

Agnès Pecolo, "Oser les cheveux blancs : une stratégie de communication à Bordeaux »,

Communication et organisation [En ligne], 40 | 2011, mis en ligne le 01 décembre 2014, consulté le 20 avril 2019. URL : http://journals.openedition.org/communicationorganisation/3618; DOI : 10.4000/ communicationorganisation.3618 


\title{
Oser les cheveux blancs : Une stratégie de communication à Bordeaux
}

\author{
Compte rendu d'entretien réalisé auprès de Michèle Walter-Canales, en charge \\ du dossier au sein de la direction de la communication.
}

Présentée dans Cap'com ${ }^{1}$ comme une des pionnières en communication senior, nous avons été à la rencontre de la direction de la communication de la mairie de Bordeaux. Relais de deux grands services dédiés aux seniors présentés comme particulièrement actifs (le pôle senior et le Centre Communal d'actions Sociales qui proposent de nombreux projets ou actions) et en charge de l'accompagnement des temps forts (comme des évènementiels seniors : la dictée des seniors ou la semaine bleue par exemple), la direction de la communication se donne pour mission de parvenir à concerner les plus de 42000 seniors bordelais à la vie de leur cité (" qu'ils s'y sentent bien »). C'est globalement l'entrée « bien vieillir » que l'on met en avant, cette idée que Bordeaux soit pour sa population senior une ville où il fait bon vivre car lui facilitant la vie, une cité capable d'accueillir des personnes vieillissantes mais pour autant « toujours actives ». L'appropriation par les seniors d'une ville que l'on veut faite pour tous est avancée. Si les " seniors » représentent pour la ville les + de 60 ans (20\% de la population bordelaise), des découpages dans la « cible » sont clairement mis en place :

- les 60-75 (traditionnellement nommés les « jeunes " seniors, grands-parents actifs, utilisateurs, consommateurs et producteurs de biens et services) ;

- les 75-85 ans (mentionnés comme les « anciens » et appelant un accompagnement spécifique pour éviter l'isolement) ;

- les + de 85 ans (les "grands aînés » qui sont en situation de fragilité avec une gestion de la dépendance grandissante).

Une population que l'on cerne par ailleurs comme complexe à toucher car hétérogène et ce, au-delà de la question de l'âge variable stricto sensu. C'est en effet plus largement les conditions de vie, loin d'être identiques qui sont évoquées, rappelant par là combien les inégalités sociales intra-générationnelles ne doivent pas être oubliées.

Par ailleurs, le positionnement souhaité est plutôt transversal dans le sens où l'on favorise et souhaite systématiser la présence d'informations destinées aux seniors au même titre que celles concernant spécifiquement l'enfance et la jeunesse, dans tous les supports de communication généralistes (guides

1. Cap'com est le réseau de la communication publique et territoriale. Dominique Mégard, "Communiquer senior, c'est l'avenir... ", Site de Cap'com, [En ligne. Paru le 2 février 2012] : http://www.cap-com.org/ actualite/2615-communiquer-senior-c-est-I-avenir.html. 
culturels par exemple), mais aussi plus thématiques tel que par exemple le guide de la rentrée scolaire qui peut concerner aussi les grands-parents, souvent baby-sitters. Cette stratégie a le mérite d'éviter une logique de différentiation de seniors particulièrement sensibles à toutes formes de ghettoïsation et ayant tendance à ne pas souhaiter s'identifier comme appartenant à la catégorie «vieux ». Acquérir le « réflexe senior » pour toute production communicationnelle est l'objectif (posé comme non encore gagné à ce jour) et si des supports spécifiques seniors sont produits (tels le guide senior ou la page Facebook senior ${ }^{2}$ ), ils ont vocation à être diffusés dans toutes les structures municipales et non simplement dans celles spécifiquement seniors (comme les clubs seniors existant dans la ville). Cette idée d'inclusion des personnes âgées dans tous les secteurs de la vie est vive et valorise les initiatives intergénérationnelles telles qu'on les repère dans les dynamiques de quartier qui mélangent de fait les âges et cycles de vie mais aussi lors de concours de collecte d'histoires et d'images de mémoires sur la ville avec des ateliers d'initiation informatique et un dialogue intergénérationnel ${ }^{3}$. Ainsi fait-on attention dans les supports de communication à diffuser des images de seniors en famille.

Dernier élément à retenir justement, la question de la représentation physique des seniors de la ville, cruciale pour un service qui porte grande importance aux visuels de sa communication. La stratégie est ici double : le local et le physique âgé assumé. Développant une politique globale d'ambassadeurs de la ville, la communication travaille avec des Bordelais qui acceptent de prêter leur image et sont les vecteurs du bien vivre à Bordeaux.

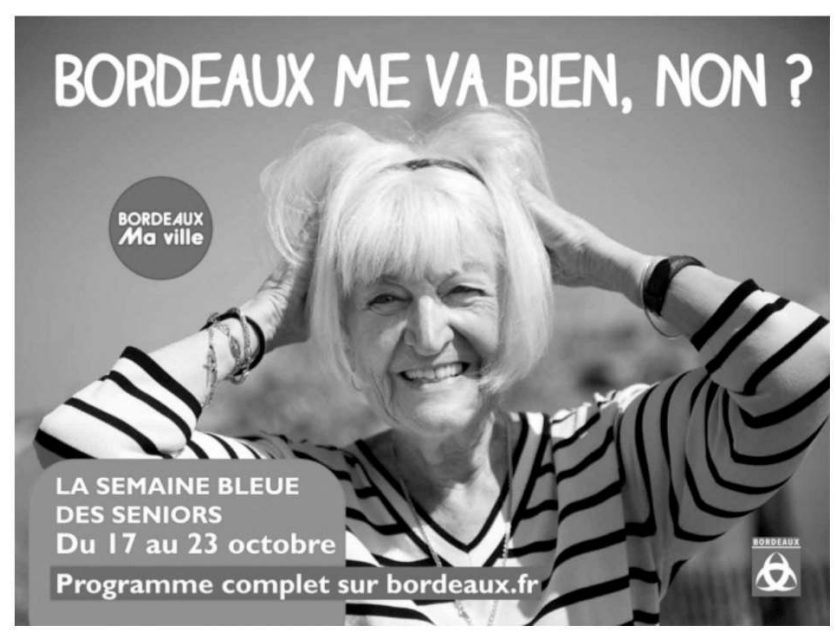

2. Facebook, page Facebook Senior : http://fr-fr.facebook.com/Bordeaux.Seniors.

3. Un blog de Bordeaux.fr, [En ligne] : raconte.bordeaux.fr. 
C'est donc une mamie bordelaise qui est l'ambassadrice plus particulièrement pour ce qui relève des communications seniors, une mamie qui déclare que "Bordeaux lui va bien " mais surtout une mamie de 80 ans qui pose et porte rides et cheveux blancs sur le devant de la scène. Le message est clair, exit les représentations de femmes bien plus jeunes que la cible visée, une pratique médiatique courante qui traduit clairement les difficultés d'assumer la vieillesse, au moins des corps.

Pour conclure, Bordeaux ne veut pas « cacher ses vieux » mais au contraire les pose comme un atout majeur, un socle pour notre société, l'enjeu étant qu'ils trouvent leur place dans une cité leur facilitant la vie. La communication prend relais en développant des campagnes puissantes, déclinées en affichages (très) grands formats sur lesquels trône leur ambassadrice senior tout en sourires... 